\section{En búsqueda de una epistemología para la investigación administrativa en América Latina}

\author{
In search of an epistemology for administrative research in \\ Latin America
}

\begin{abstract}
RESUMEN
La Administración es una ciencia que contiene teorías, principios, tecnologías y prácticas. Como campo de investigación, tiene dos grandes problemas: primero, que ha sido impelida tanto por las universidades como por los organismos de promoción del desarrollo de la ciencia al uso de la epistemología positivista o cuantitativa, tal y como si fuera una ciencia natural; segundo toca estudiar la aplicabilidad a todo lo descubierto y creado en los países desarrollados a la realidad de Latinoamérica, sobre todo porque la realidad social de este subcontinente tiene su propias peculiaridades. En estos esfuerzos puede ayudar mucho la Antropología Social, con el uso de sus métodos de investigación esencialmente cualitativos.
\end{abstract}

Palabras claves: Epistemología; Antropología Social; Administración; Prácticas Gerenciales.

\begin{abstract}
The Administration is a science that contains theories, principles, technologies and practices. As a field of research, it has two major problems. First, it has been impelled both by universities and by organizations promoting the development of science to the use of positivist or quantitative epistemology, as if it were a natural science. Second is to study the applicability to everything discovered and created in developed countries to the reality of Latin America, especially because the social reality of this subcontinent has its own peculiarities. Social Anthropology can help a lot in these efforts, with the use of its essentially qualitative research methods.
\end{abstract}

Keywords: Epistemology; Social Anthropology; Administration; Management Practices.

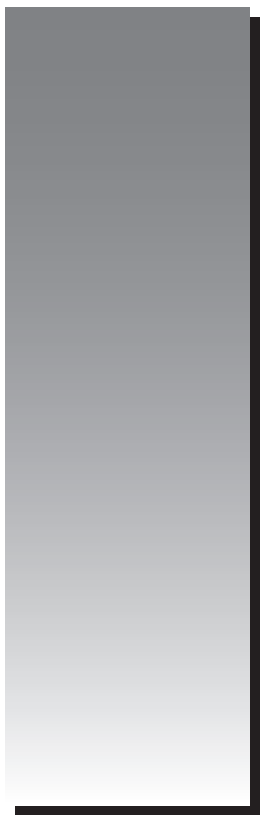

Augusto Hidalgo Sánchez

ahidalgos@unmsm.edu.pe

Universidad Nacional Mayor de San Marcos, Facultad de Ciencias Administrativas. Lima, Perú 


\section{INTRODUCCIÓN}

Cuando un profesional en Administración latinoamericano ha laborado por algunos años en una o varias organizaciones del hemisferio norte, y luego se regresa a su país para continuar su vida como gerente, inevitablemente acaba construyendo un edificio mental de conclusiones administrativas paralelas, y por qué no decir hasta cierto punto contradictorias. Concluirá que existen muchas semejanzas entre ambas realidades laborales, pero que, además, que existen profundas diferencias. Las semejanzas se ubican en el campo tecnológico, y las diferencias en la función gerencial en ambos espacios. Es decir, las organizaciones son gerenciadas de manera distinta, a distintas velocidades, en hemisferios diferentes.

Esta dualidad de realidades gerenciales debería conducir a una intensa preocupación en la actividad de enseñanza gerencial en nuestros países, pero verificamos que no es así, ya que nuestra enseñanza se basa en el uso exclusivo de literatura norteamericana, y europea en menor grado, y muy poca o ninguna latinoamericana. Nuestras universidades e instituciones académicas deberían hacerse cada día la pregunta: ¿es la teoría gerencial de aplicación universal como lo es la Física o la Biología? Hay que precisar que cuando hablamos de teorías gerenciales nos referimos a aquellas que tienen que ver con la dirección del comportamiento humano y la toma de decisiones en las organizaciones. Aquí excluimos a los textos de tecnología gerencial como: Finanzas, Logística, Marketing, Costos, Presupuestos, Auditoría, Publicidad, Selección de Recursos Humanos, etc. las cuales sin lugar a dudas son conocimientos sociológicamente neutros, y por definición, son de aplicación universal.

La descreencia y el eclecticismo de Henry J. Mintzberg en la efectividad de la enseñanza en aulas de las habilidades para la gerencia de las personas, (Mintzberg, 2005) agudizan nuestra duda y necesidad de plantear esta interrogante desde una perspectiva sociológica y antropológica. Para América Latina, una región económica, tecnológica y culturalmente distante de Norteamérica, Europa o Japón, parece urgente que nos pongamos a revisar dichas teorías gerenciales desde una perspectiva nativa $\mathrm{y}$, si es posible, con ayuda de la epistemología antropológica.

\section{La Naturaleza de la Epistemología de la Administración}

La naturaleza ontológica y epistemológica de la Administración ha sido bastante debatida y lo sigue siendo. Tal vez esto es parte de la maduración del juvenil conocimiento administrativo. Debemos partir de reconocer que las teorías administrativas son todas jóvenes, en pleno proceso de crecimiento, no tienen más de cien años, a diferencia de la Medicina, la Física o el Derecho que tienen milenios. Probablemente, esa falta de añejamiento sea la causa de que hasta la fecha no exista claridad sobre la naturaleza del conocimiento ni de la epistemología administrativa.

Las dudas están sembradas por todos lados. Por ejemplo, los filósofos de la ciencia, como Mario Bunge, no creen que la Administración siquiera alcance la talla de ciencia (Bunge, 2014). Por su parte Karl Popper, un poco más generoso y vago, nos obsequia una ilusión general al expresar que los gobiernos en vez de búsqueda de utopías, deben desarrollar medidas concretas de gobierno que él llama "Ingeniería Social" (Popper, 1985), o Gerencia, a la que le otorga un valor fáctico, aunque nunca la llame ciencia, tampoco.

No obstante, independientemente de lo que opinen los filósofos, los investigadores o los especuladores del conocimiento, la humanidad sigue evolucionando, las organizaciones en el mundo continúan creciendo en tamaño y complejidad. Hace un siglo una organización gigante tenía unos 30,000 trabajadores, hoy esa sería una organización mediana, ya que las gigantes sobrepasan el millón de trabajadores, como es el caso de la tienda de departamentos Walmart. Esto ha hecho que la Administración haya tenido que desarrollar apuradamente, y a jalones, un cuerpo de conocimientos donde conviven los de tipo científico y los no científicos, y que de manera convergente la hayan convertido sin duda alguna en una disciplina central y en una profesión de elevado prestigio en la sociedad moderna. La Administración es hoy el oficio que permite operar a la mayoría absoluta de organizaciones productoras de bienes y servicios locales o mundiales como acróbatas o relojes 
sincronizados a velocidades crecientes, en estructuras multinacionales, que corren de uno a otro lado del planeta, con el magnífico resultado de ofrecer, de manera creciente, los bienes y servicios que requieren unos seis mil millones de habitantes de este planeta. Este oficio provee de trabajo remunerado a millones de directivos, gerentes, administradores, apoderados, supervisores, caporales, técnicos y auxiliares en el mundo.

Tal vez sea la complejidad de las organizaciones y sus entornos, es decir la naturaleza del objeto de la Administración la causa de este entrevero y oscuridad epistemológica. En un siglo de existencia, la Administración ha construido un enorme cuerpo de conocimiento, nacido de un sinnúmero de fuentes de diversas disciplinas. Por esta razón, algunos teóricos la llaman ciencia de segundo orden (Jiménez, 1981). Y en su evolución ha ido adoptando y adaptando conocimientos de otras disciplinas tanto cuantitativos como cualitativos al punto que en la actualidad tampoco tiene límites precisos. No se sabe, por ejemplo, dónde acaba la administración y dónde comienza la Ingeniería, tampoco el Derecho, la Sociología, ni siquiera la Psicología.

Sin embargo, a pesar de esta colosal complejidad de la Administración que requiere de una gran apertura intelectual, social y académica, muchos reglamentos de investigación académica de diversas universidades latinoamericanas, de manera ciega y a fardo cerrado admiten solamente el enfoque epistemológico de la ciencia natural, es decir, sin considerar que podría haber otros paradigmas que permitan comprender y aprehender el Management. El corazón de dichos reglamentos establece normativamente, valga el pleonasmo, que la investigación y la producción de conocimientos en Administración debe seguir el protocolo probatorio, positivista o cuantitativo. Y es precisamente este mismo enfoque que los organismos de promoción de la ciencia de nuestros países latinoamericanos han adoptado para todas las disciplinas. Es decir, si los investigadores en Administración desean producir conocimiento o llegar a ser considerados investigadores científicos, entonces deben seguir la misma metodología de las ciencias naturales: Biología, Química o Física, que siguen un protocolo probatorio, establecido en el modelo: PROBLEMA -
HIPOTESIS - OBJETIVOS - ANÁLISIS ESTADÍSTICO - PRUEBA DE HIPÓTESIS.

Tres argumentos contundentes rebaten las posiciones positivistas con respecto a la Administración.

Primero, parece que existiera una voluntad de ignorancia selectiva con relación a lo que piensan los estudiosos de las ciencias sociales, por lo menos desde la década de los 70's. Friederich von Hayek en su discurso de recepción del Premio Nóbel, el mismo que ha sido denominado "Pretensión del Conocimiento" (Hayek, 1974) denunció a aquellos que basándose en el uso de las técnicas cuantitativas pretenden reducir la realidad en dos o pocas más variables, con la intención de crear una función matemática simple (de primer grado). Advirtió que la realidad en la Economía es demasiado compleja como para que una variable dependiente pueda ser explicada en función de una o pocas variables independientes. Argumentaba que ese procedimiento reduccionista constituye un abuso imperdonable, y muy por el contrario, lo que corresponde hacer es tratar de comprender la complejidad de los fenómenos sociales construyendo modelos más o menos racionales.

Segundo, para una disciplina como la Administración, cuyas mayores contribuciones científicas se encuentran en el campo cualitativo, algo anda mal con esa exigencia de naturaleza cuantitativa. Robert McNamara, ex ministro de defensa de los Estados Unidos en las épocas de Kennedy y Johnson y quien dirigió la Guerra de Vietnam en los 60’s llegó a la convicción de que en dirección considerar solamente las variables cuantitativas es de un riesgo enorme. En su nombre precisamente se ha consolidado una nueva teoría administrativa denominada "Falacia de McNamara”, (McGrath \& Bates, 2013). Advirtió que los gerentes tradicionales basan su ejecutoria y pensamiento gerencial en cuatro sencillas premisas:

1. Medir aquello que puede ser medido, sobre todo de lo evidente,

2. Descartar aquello que no puede ser medido,

3. Asumir que aquello que no puede ser medido no es importante, y finalmente 
4. Asumir que aquello que no puede ser medido no existe.

McNamara se dio cuenta y denunció esta forma de pensar por falaz y descriteriada, su inutilidad se demostró en la errada gestión militar de la Guerra de Vietnam, cuando los generales americanos medían su éxito por el balance de número de muertos en los dos bandos. Al final de la guerra hubo 58,000 norteamericanos muertos y un millón y medio de vietnamitas. Pero, a pesar de esos números exitosos, la historia ha registrado que quien clamó por la paz, mejor dicho, quien perdió la guerra, fueron los Estados Unidos en 1975. Los vietnamitas, a pesar de su enorme cantidad de muertos, consideraban que estaban recién en etapa de afinamiento de los métodos de guerra y preparándose para contiendas de fondo.

Tercero, el error del sesgo cuantitativo en investigación en Administración resulta también fácilmente desnudado y ridiculizado por el simple hecho tangible de que los activos más importantes en las organizaciones, es decir sus capacidades y ventajas competitivas, no son las reservas minerales, ni financieras, ni la tecnología, ni otros elementos fácilmente medibles o adquiribles, sino son su reputación, su posicionamiento y sobre todo el conjunto de rutinas generadoras de eficiencia, la capacidad de logro de objetivos de su personal, la innovación, la responsabilidad social, es decir, con todo aquello que está asociado con el nivel de conocimiento colectivo, con la cultura organizacional, con los valores, con la actitud y con la voluntad de los trabajadores o lo que se denomina ahora Capital Social. Desafortunadamente, nada de este capital puede ser medido con números. Estos conceptos no se pueden medir en la empresa con ningún sistema de indicadores o de tableros. Es mucho más complejo y sutil que eso. Tal vez nos falta reflexionar un poco más sobre la definición de bienes de una empresa, en el diálogo entre Sócrates y Critóbulo descrito por Jenofonte. (Jenofonte, 1990)

Correspondientemente, así como no es posible convertir la gerencia integral en un simple asunto de ratios o indicadores, a pesar de los múltiples esfuerzos de autores inteligentes como Kaplan y Norton en su "Balanced Scorecard" (Kaplan \& Norton, 1996), tampoco es razonable cuantificar todo como Fred David con su método de planeamiento estratégico (David, 2003). Asimismo, tampoco es posible investigar asuntos de gerencia y convertirlos en hipótesis que puedan ser comprobadas con facilidad usando estadística inferencial.

Ni siquiera la Economía pura ha nacido de hipótesis probadas estadísticamente, tal es el caso del modelo de economía de mercado de Adam Smith que fue diseñada varias décadas antes que se formulara siquiera la distribución normal de probabilidades de Gauss o el cálculo de regresión múltiple. La razón de fondo de esto reside en el hecho que en el fondo de toda teoría de negocios siempre habrá un componente cualitativo, ya que todas ellas se refieren o tienen como objetivo final la conducta humana sea como trabajador, como jefe, como cliente, como consumidor, como agente económico en general. Y este agente tiene voluntad, tiene percepción, tiene valores, tiene creencias, tiene conocimientos, tiene afectividades, es decir es un ser humano.

La complejidad epistemológica de la Administración la podremos percibir mejor si observamos su evolución. Las primeras teorías o escuelas de la Administración surgidas a comienzos del siglo XX se volcaron a construir cuerpos de conocimientos de tipo preceptivo (principios de la administración) "sobre la base de un razonamiento que podría ser llamado de metafísica normativa" (Hidalgo, 2013). El resultado fue un conjunto de reglas necesarias en una organización para mejorar su eficacia y eficiencia. Las teorías de "Principios de la Administración Científica" de Frederick Winslow Taylor (Taylor, 1987) y la de "Administración Industrial y General" de Henri Fayol, (Fayol, 1987), son el mejor testimonio.

Unas dos décadas después de la aplicación de las recetas taylorianas, emergieron efectos disfuncionales, a lo que se acompañó del crecimiento interminable del tamaño de las organizaciones. Esto urgió de un nuevo método de estudio y de formulación de la teoría administrativa. Aquí es cuando hacen su arribo los métodos naturalistas, cualitativos, la investigación participativa, que habían tenido éxito en la disciplina antropológica desde cerca de un siglo. 
Precisamente, la primera investigación académica, a cargo de la Universidad de Harvard y encargada al profesor Elton Mayo y que duró cinco años, confirmó que la cultura organizacional, es decir ese conjunto de significados, símbolos, códigos, valores, compartidos tienen un efecto firme sobre las motivaciones y logros de los trabajadores. Por primera vez se comprobó que el comportamiento de los trabajadores no es resultado solamente de estímulos materiales o de las condiciones de trabajo. Que el trabajador no se comporta de acuerdo a una simple ecuación de regresión simple o de causa-efecto. Esto abrió en los años 30's un universo nuevo a la investigación en Administración: el estudio del comportamiento humano en las organizaciones.

Pero, no obstante este paso evolutivo y que el estudio de Hawthorne realizado por Mayo se hizo en una fábrica norteamericana, es decir en un medio social y económico específico, así como se han realizado infinidad de otros estudios del mismo orden, la aplicación de la ciencia administrativa en los países latinoamericanos, y tal vez en el resto del mundo, parten de la premisa más o menos aceptada que el ser humano es universal, que tiene un conjunto de conductas iguales en todos lados, es decir, que los principios administrativos serían de aplicabilidad universal.

Justamente en este principio se basa la enseñanza de la Administración en todos lados en América Latina, hemos tomado las teorías, principios o técnicas generadas en Estados Unidos y Europa como aplicables al 100\% en nuestro medio, es decir las hemos tomado a fardo cerrado, sin reflexionar mayormente en su contenido, ¿qué diría René Descartes?, pues que mínimamente hay que dudar metódicamente (Descartes, 2014).

Henry Mintzberg ha tocado una tremenda campana de alerta, cuando advierte que las habilidades del gerente son dos: por un lado, manejo de tecnologías administrativas, y por otro el talento gerencial (Mintzberg, 2005). Las técnicas son neutras y muy fáciles de aprender y aplicar indistintamente de las circunstancias organizacionales, pero el talento para gerenciar grupos humanos requiere del desarrollo lento de otros tipos de habilidades, menos escolásticas y no tan explícitas.
Ahora, si asumimos que los seres humanos tenemos diferencias etnográficas, culturales, sociales, es decir antropológicas, entonces, tenemos el derecho legítimo a dudar del conocimiento que se vende como universal o de talla única, tenemos derecho a sospechar de que los principios o teorías de la Administración descubiertos o formulados en cierto contexto social, político, de idiosincrasia, de desarrollo industrial, para ciertos seres humanos de ciertas nacionalidades avanzadas, sean norteamericanos, ingleses o franceses, pues, no son universales. Tenemos derecho a cuestionar que algo de dicha ciencia en su estado actual necesita ser ajustada para su plena aplicación en los países menos desarrollados y con una historia diferente como la latinoamericana.

Por otro lado, si aceptamos que la Antropología nos ayuda a comprender las diferencias entre los seres humanos, entre las diversas comunidades, ente diferentes idiosincrasias, entonces debemos preguntarnos, ¿qué sabemos de la antropología del latinoamericano?, ¿qué es un latino?, ¿en qué se diferencia, digamos, de un francés, o de un japonés?, ¿tienen los latinos mayor tendencia a ser ingeniosos, a ser informales, a ser leales o a ser ingratos, a ser evasores de la ley, a ser tolerantes o a ser violentos en el trabajo, a ser corruptos, a leer las órdenes con detenimiento, a cumplir las órdenes de sus jefes con total disciplina? Ahora profundizando un poquito la pregunta ien qué se diferencia un caribeño de un altoandino o de un selvático? por poner sobre la mesa sólo algunas dudas de fondo.

Richard Webb economista e investigador social peruano, por muchos años presidente del Banco Central de Reserva del Perú, y líder de opinión ha cuestionado la naturaleza general de la investigación universitaria en América Latina (Webb, 2015). Sostiene que nuestras universidades son tercas en perseguir como un fantasma a la ciencia dura, en querer ponerse a la altura de las universidades top de los Estados Unidos, cuyos presupuestos anuales sobrepasan a las nuestras en decenas, sino en cientos de veces. Esa inquietud por competir con ellas es deseable, pero no es posible, y desde su perspectiva incluso es accesoria cuando dice que "... el conocimiento más crítico que necesitamos no son las leyes de la llamada ciencia pura, de la 
química, la física y la biología, sino los secretos de la vida social". Y agrega que:

[...] muchísimo más productivo para la vida nacional sería entender las deficiencias en el comportamiento humano. ¿Por qué tenemos niveles tan altos de informalidad? ¿De corrupción? ¿De delincuencia? ¿Qué traumas de niñez, quizá, nos impiden respetar las leyes del tránsito? ¿0 las normas cívicas de las colas? ¿Qué hay en nuestros genes, o en nuestra historia, que nos ha vuelto tránsfugas campeones, tan irrespetuosos de las lealtades institucionales y políticas?

$\mathrm{Al}$ respecto es bueno por ejemplo echar un vistazo a las cifras de Proética, una ONG (Organización No Gubernamental) dedicada a estudiar la corrupción en el Perú, que estima que los efectos económicos de la corrupción podrían ascender hasta el 5\% del PBI, unos 10 mil millones de dólares: más de seis veces el presupuesto de todos los programas sociales para este año (Webb, 2016).

No sería entonces atrevido que tal vez empecemos por analizar el sustento epistemológico de la investigación de Herrnstein y Murray, (Herrnstein y Murray, 2004), dos destacados profesores de la Universidad de Harvard quienes en el año 1994 publicaron un polémico libro llamado "La Curva Normal", en el cual sostienen que las razas negra y latina en los Estados Unidos tienen menor coeficiente intelectual que los blancos o asiáticos, y por deducción seguramente explica nuestro subdesarrollo como países. Si confirmamos los resultados de dicha investigación tal vez nos llevaría a reflexionar sobre la verdadera capacidad intelectual de nuestros antepasados pre-incas, o mayas quienes crearon el Estado de manera independiente y construyeron en su tiempo sociedades avanzadas, exactamente tal como lo hicieron también por su cuenta los chinos, los hindúes, los egipcios, y los mesopotamios, cuando los europeos de raza blanca aún eran pueblos bárbaros.

Ahora si nos enfocamos en la gerencia de las organizaciones latinoamericanas, las preguntas esenciales se deberían referir a la existencia de diferencias con extranjeros, o entre los propios latinos, entonces cabría preguntar, ¿cuáles son los principios apropiados para ad- ministrar una organización latinoamericana a diferencia de los aplicados a una organización extranjera?, ¿en qué deberían variar los principios o prácticas que se deberían usar para administrar una empresa minera en los Andes de Perú o para una organización portuaria en El Callao, con respecto a otra de China?, ¿habrá diferencias entre ambos?, ¿en qué aspectos y en qué grados existen diferencias? ¿Son sustanciales o marginales dichas diferencias?

Sin embargo, una reflexión crucial sobre la validez universal de las teorías administrativas debería partir del hecho que la práctica gerencial de las organizaciones latinoamericanas es tan buena como en cualquier parte del orbe. Esa es la razón de su éxito. Alguien con razón podrá preguntar entonces ¿y cómo es que si el objeto de la administración (seres humanos) es diferente las empresas latinoamericanas alcanzan el éxito sin que exista una ciencia consolidada de la administración de empresas latinoamericanas? A modo de hipótesis se podría plantear que es debido por un lado a la aplicación masiva de tecnología administrativa, incluida la informática que es universal, y por otro, a la intuición y habilidad de los gerentes locales, mas no así a la existencia de una ciencia gerencial universal, concebida, gestada y nacida en y para las organizaciones de los países del primer mundo.

Es decir, es posible que su éxito se deba no exactamente a razones académicas o científicas, es decir a la actual ciencia gerencial mundial, ni siquiera es mérito de alguna universidad o escuela de enseñanza de Administración. Sino más bien, como sostiene Mintzberg es debido a que los administradores a cargo de las organizaciones locales tienen el talento suficiente y han desarrollado la agudeza para comprender bien la fenomenología del trabajador local y del entorno organizacional local y aplicar un enfoque o estilo de administración llamemos particular o específico (Goosen, 2004), Consecuentemente, han sido capaces de bosquejar, ajustar o ensayar principios, modelos o reglas exitosas propias, que por ensayo y error han acabado acertando y arrojando un conjunto de prácticas administrativas exitosas en este medio. A eso podríamos llamar prácticas gerenciales particulares.

Pero ¿cuánto de dicha práctica gerencial particular ha sido registrada? Generalmente las 
prácticas exitosas (así como las no exitosas) no se registran, eso queda en el know-how de las organizaciones, en su cultura, en su capacidad competitiva y en las habilidades directivas de sus gerentes. Pero, eso no constituye ciencia, porque no existe ciencia privada. La ciencia es aquel cuerpo de conocimiento que ha pasado por el escrutinio público, por debate. La ciencia es abierta por definición, no es arcana (así, por ejemplo, el conocimiento interno que maneja la secta de los rosacruces, u otras sectas masónicas, no califica como ciencia, precisamente por ser privada). Si descubrimos, compartimos, debatimos sobre lo que constituyen esas prácticas gerenciales particulares exitosas, entonces estaremos comenzando a hacer ciencia.

Es decir, por lo menos teóricamente, si las preguntas anteriores sobre las prácticas gerenciales en América Latina son respondidas de manera explícita, si las preguntas y las respuestas las llevamos al mundo académico, tal vez podríamos mejorar la enseñanza de la Administración, y seguramente, el desempeño de las organizaciones latinoamericanas sería mejor. Recordemos que "Administración Industrial y General" de Henri Fayol no buscó pretenciosamente (como diría von Hayek) crear una nueva ciencia, sino humildemente escribir un conjunto ordenado de prácticas y observaciones que podrían ayudar a otras personas a administrar organizaciones industriales en el medio europeo. Pues, hay que regresar a este origen con espíritu de esperanza del pionero.

En esta tarea de comprender la esencia de la Administración de las organizaciones latinoamericanas toca a la academia (universidades) hacer, por un lado, un levantamiento de las prácticas gerenciales particulares en nuestras organizaciones y construir un mapa de las características antropológicas del ejercicio gerencial en nuestro territorio. Y lo que es más, esta pareciera ser una tarea que requiere sea realizada no solamente una vez sino periódicamente, digamos cada 10 años. Porque lo más seguro es que factores exógenos gigantescos, como la globalización económica, tecnológica y cultural, con seguridad que están afectando no sólo entorno (clientes, competidores, proveedores, gobierno), sino la propia conducta de los latinoamericanos.
Tenemos que partir del reconocimiento que no tenemos la definición de qué es un latinoamericano, no sabemos qué aspira, qué prefiere, qué detesta, qué teme, qué le hace soñar, qué nivel de convicción religiosa tiene, ni cuál es su escala de valores. Tenemos abundantes sospechas de ellas, pero no tenemos precisiones. Nos urge la definición de qué es un latinoamericano, de su fenomenología antropológica. Aquí nos puede dar una mano la ciencia de la Antropología.

\section{La Administración y la Antropología.}

Los conceptos de Antropología de Negocios, Antropología Corporativa, Etnografía de Negocios, Antropología del Trabajo, Antropología aplicada a la Industria o Antropología Industrial emergieron en el lenguaje académico y se volvieron comunes en Estados Unidos en los años 80's.

La Antropología es una ciencia social que estudia el comportamiento humano y sus culturas, el ambiente social en el cual viven las personas y el impacto de este ambiente sobre los sentimientos, actitudes y comportamiento. La Administración por su parte, es una disciplina social dedicada a comprender, a predecir y a influenciar el comportamiento de las personas para que la misma logre determinados fines dentro de una organización. La naturaleza de ambas es opuesta, mientras que la Antropología es una ciencia especulativa, de análisis y de comprensión de la realidad humana, la Administración es esencialmente una disciplina activa, ejecutiva, desempeñada en organizaciones con el propósito práctico de lograr objetivos, es decir es purposive como dicen los anglosajones.

\section{Algunas pistas de las diferencias antro- pológicas de los latinoamericanos.}

El latinoamericano, es producto de un largo proceso de mestizaje indo-afro-europeo. Los casos de Perú y México se parecen mucho más debido a que ambas naciones tenían una importante cultura y población aborigen, por lo que incluso racialmente son muy parecidos. Tienen grupos aún con cosmovisiones y patrones de conducta influenciados por sus culturas precolombinas, pero tienen también otros grupos, llamemos criollos, quienes mantienen rezagos del modo de actuar que usaron para vivir o sobrevivir en 
el largo periodo virreinal y de nuestras repúblicas caóticas, estos rezagos se llaman viveza criolla, que contiene una alta carga de comportamiento organizacional y social indisciplinado o inclusive anómico. El problema es que no existen precisiones respecto de los segmentos de la población o del grado en que están afectados por esas conductas.

Por otro lado, el Perú, por tomar un caso, es un país en vías de desarrollo, su PBI está por los US\$13,500. El país contiene zonas de ciudades propias de país desarrollado, pero también tiene amplios sectores no desarrollados, incluso pequeñas poblaciones en estado primitivo o no contactado. Emile Durkheim publicó su tesis doctoral sobre la división social del trabajo en Francia, en la época en que dicho país ingresaba a la era de la industrialización, cuando se producía una migración campo-ciudad y una reconfiguración de las relaciones sociales de la sociedad francesa. Durkheim incorporó los conceptos de solidaridad mecánica y solidaridad orgánica para distinguir los tipos de lazos que explicaban la cohesión social de las personas que vivían por un lado en el campo y por otro lado en la ciudad. Pareciera que una investigación elemental debe tratar de comprender qué tipo de lazos unen a los latinoamericanos en sus diferentes estratos sociales. Preguntarnos en qué temas es cohesionado, por ejemplo, en sus valores patrios, o familiares, o en qué grado es disciplinado o respetuoso de la ley o en qué grado es anómico.

Tal vez, por ejemplo, aún nos falta comprender al latino básico, tal como Malinowsky comprendió al aborigen de Oceanía a través de su trilogía Magia-Ciencia-Religión (Malinowsky, 1948). Necesitamos saber cuánta magia, cuánta ciencia o cuánta religión pone un latinoamericano en su cosmovisión y consecuentemente en su comportamiento organizacional.

\section{Algunas interrogantes específicas sobre la universalidad de las teorías gerencia- les a la luz del enfoque antropológico.}

Hagamos un ejercicio de cuestionamiento teórico, es decir hipotético acerca de la vigencia de las teorías administrativas tradicionales más conocidas a la gerencia en América Latina a la luz de las evidentes diferencias antropológicas.

\section{Teoría de Administración Clásica de Fayol.}

Henri Fayol, ejecutivo francés, reflexionó profundamente sobre su larga experiencia gerencial y usando digamos una especie de metafísica normativa le llevó a formular las funciones de la empresa, las funciones de la administración y 14 principios (mandamientos) administrativos. Surge entonces la pregunta ¿si Henri Fayol hubiera realizado su trabajo en Perú, habría formulado aquellos 14 principios?, ¿no habría tal vez agregado otro por ejemplo así: "Cumplimiento honesto de la tarea encargada" (es decir ejecución del trabajo sin viveza criolla, ni picardía).

\section{Teoría de las Relaciones Humanas de Mayo.}

Elton Mayo, profesor de la Universidad de Harvard, desarrolló los estudios de Hawthorne descubrió que en toda organización coexisten dos universos paralelos pero interrelacionados, uno formal y otro informal. Aquel formal se relaciona con las finalidades de la organización y el informal con los objetivos y metas de cada individuo. Conociendo la naturaleza demográfica de los latinos que se encuentran en medio del recorrido del camino de evolución entre sociedad tradicional y sociedad moderna, sería tal vez apropiado preguntar si en una organización integrada por trabajadores y gerentes latinoamericanos ¿cuál es el peso o ponderación de la organización informal en ella?

\section{Teoría de la Autoridad de Max Weber.}

Max Weber, sociólogo alemán, se preguntó ¿por qué las personas obedecen? Y en su respuesta delineó tres fuentes de autoridad: tradicional, carismática y racional. Precisamente a esta última la identificó como la verdadera fuente de la autoridad en las organizaciones en una sociedad civilizada, que tiene por base la constitución política y su sistema de leyes y reglamentos. De nuevo, cabe preguntar según el parágrafo anterior chan internalizado los latinoamericanos a la autoridad racional como la fuente más apropiada de donde emana la autoridad en las organizaciones?, ¿por qué existe tanto amiguismo o nepotismo en las relaciones laborales?, ¿en cuánto afecta su condición de país en vías de desarrollo y de país con innume- 
rables agresiones al sistema legal debido a los múltiples golpes de estado y a la debilidad de sus sistemas de administración de justicia?

\section{Teoría de la Escala de Necesidades de Maslow.}

Abraham Maslow, psiquiatra norteamericano, explicó el motor que conduce a las personas a actuar, sea en su vida privada o en su trabajo en organizaciones. A ese motor denominó Escala de las Necesidades Humanas (Maslow, 2012). Si él hubiera realizado su investigación en América Latina, ¿hubiera formulado la misma escala de necesidades de cinco niveles? Es decir, habiendo esta teoría sido formulada en un país desarrollado, ¿tiene aplicabilidad universal, sobre otras razas, países o circunstancias diferentes de su génesis? Estas preguntas caben porque el autor fue cauto y modesto, al estilo de Fayol, precisamente el libro que publica esta escala se titula en idioma inglés "A Theory of Human Motivation" (Una Teoría de la Motivación Humana).

Por ejemplo, para un poblador aymara de las alturas del Lago Titicaca, quien mantiene aún un profundo enraizamiento con su cultura aborigen, es decir vive y mantiene una cosmovisión milenaria dogmática, ¿se aplicará la mencionada escala de necesidades? Lo mismo podríamos preguntarnos en forma un poco más global ¿para un musulmán profundamente religioso, se aplicará del mismo modo la mencionada escala? Tal vez la Antropología Social nos permita una luz para confirmar que Maslow efectivamente ha tenido la preclaridad de ingresar al fondo del alma del ser humano es decir de abarcar a poblaciones a las que tal vez ni él mismo había conocido.

\section{Teorías del Planeamiento Estratégico.}

Un conjunto de teorías surgidas desde los años 60 's han convergido en una superherramienta gerencial llamada "Planeamiento Estratégico" o "Administración Estratégica". David y otros de sus principales representantes seguramente la han compilado y estructurado como una ayuda tecnológica gerencial (David, 2003); sin embargo, desde los años 90`s, ha sido divulgada como el mejor camino, sino el único, para llegar a ser competitivo local o internacionalmente. ¿Es eso cierto?, ¿alguna compañía la ha utilizado en ese sentido y ha logrado su objetivo? Aparte de las diferencias de entorno que seguramente nuestras compañías latinoamericanas tienen, ¿no será que algunas de las debilidades de la teoría del planeamiento estratégico radiquen más bien en sus protagonistas humanos?

\section{Las ideas antisistema de Mintzberg.}

Henry Mintzberg, economista norteamericano y autor de una innumerable cantidad de investigaciones y ensayos en el campo de la gerencia, ha cuestionado radicalmente la aplicabilidad de las teorías gerenciales, no solamente por las diferentes idiosincrasias o culturas de las organizaciones, sino en general y de manera casi nihilista, debido a la esencia misma de inaplicabilidad de dichas teorías. Consecuentemente, para Mintzberg es imposible enseñar a gerenciar, y que la realidad es infinitamente más compleja que cualquier teoría al respecto. Más bien que cada gerente llega a ser exitoso es debido a su capacidad de comprender el fenómeno que administra, talento que por ensayo y error, el tratamiento y solución de cada problema va siendo sintetizado y poco a poco convertido en prácticas gerenciales, propias, únicas e irrepetibles de la organización donde las aplica con éxito. En este escenario, las teorías gerenciales estudiadas en las aulas de maestrías en el mundo tienen poco que ver con el éxito. De un modo radical, él considera que las escuelas de MBA's en el mundo han fracasado.

\section{Áreas en los que puede ayudar la Antro- pología Social a la Epistemología de la Administración.}

Necesitamos de la Antropología Social tanto por sus conceptos como por su metodología. Esta ciencia tiene por tanto un enorme potencial para el desarrollo de investigación multidisciplinaria en el campo de la gerencia tanto conceptualmente como metodológicamente. Tiene la virtud epistemológica de usar de gran manera el método de la inmersión etnográfica y la virtud ontológica tanto de describir de manera precisa los contextos como de comprender de manera correcta cómo esos contextos son interpretados y experimentados por los participantes. La Antropología Social puede ser utilizada y desarrollada en todos los campos de la gerencia, muy en especial del desarrollo de los conceptos de cultura organizacional, diferen- 
cias etnográficas, globalización, velocidad del cambio, diferencias o semejanzas de puntos de vista.

En resumen, la Antropología social podría apoyar a la Administración en tres campos:

- Enfoque en la Cultura. Es decir, significados, tanto significados compartidos como significados conflictivos y consecuentemente el origen del conflictivo.

- Enfoque en la Crítica. Superar la idea generalizada en Administración de dar por sentado que lo que sostiene tal o cual teoría o principio administrativo es aplicable de manera universal, como los principios de la Física. Todo hace presumir que en Administración hay ciencia grande es decir principios universales, y hay ciencia chica, es decir principios que se aplican a poblaciones menores.

- Enfoque en el Cambio. Ciertas ideas antropológicas subyacentes constituyen condiciones pre establecidas para el cambio, tanto a favor como en contra. Cómo construir la actitud de apertura, de eliminar dilemas improductivos, de enseñar las ventajas del cambio, etc. Son algunos de los tantos problemas asociados con el manejo del cambio organizacional. Esta investigación puede resultar de inmensa importancia en países como los nuestros conocidos por su incapacidad para adaptar tecnología ya existente en países más desarrollados.

\section{La Epistemología Antropológica de apo- yo a la investigación administrativa.}

La Antropología Social usa esencialmente el método de investigación participativa o llamado también de inmersión etnográfica. Este método implica desplegar una apreciable cantidad de tiempo en trabajo de campo, conviviendo con las personas objeto de estudio para ganar una comprensión profunda de los individuos y de la organización bajo estudio. Esto implica analizar diariamente los procedimientos, las reglas, las decisiones, los significados, las interrelaciones y las comunicaciones en una organización en relación a las actitudes de las personas que trabajan en ella, a sus conocimientos, a sus ha- bilidades y a sus competencias, en general. Aquí no se usan sino accidentalmente los métodos estadísticos, en su reemplazo, lo que predomina es el método de casos y la formulación de modelos explícitos sobre la base de propuestas heurísticas.

\section{Casos de investigación cualitativa con apoyo de la epistemología antropológica.}

Existen tres áreas específicas donde la Antropología Social podría ser de gran utilidad a la investigación administrativa en América Latina:

\section{Estudio de la Gerencia del Conocimiento.}

En este campo el uso de los métodos antropológicos resulta crucial debido a que la inteligencia competitiva se convierte cada vez más en capacidad competitiva única. El conocimiento organizacional es reconocido cada vez más como un activo organizacional clave. Este activo no tiene posibilidad de plagio, y crece de acuerdo con procesos de optimización de la inteligencia interna de la organización. Un sistema de Gerencia del Conocimiento debe ser capaz de organizar los activos intelectuales de una organización. Eso incluye información, experiencia corporativa y conocimiento tácito de los empleados. Sus esfuerzos se deben enfocar mayormente en los objetivos organizacionales, tales como: desempeño mejorado, desarrollo de una ventaja competitiva, innovación, participación de lecciones aprendidas, y mejoramiento continuo de la organización. Los conocimientos obtenidos por el método antropológico pueden ser combinados con aquellos propios de la Psicología, Teoría Organizacional, y Modelamiento de la Información.

\section{Estudio de la Lealtad del Cliente y el Mo- delo de Comportamiento del Consumidor}

Desde hace unos 15 años, las empresas latinoamericanas modernas, sobre todo aquellas relacionadas con el retail o tiendas de departamentos, han implementado sistemas de lealtad de los clientes (CRM - Customer Relationship Management) que les ha permitido capturar e ir acumulando una colosal cantidad de datos de compras de los clientes. Toca preguntar ¿cómo van a procesar o cómo están procesando estos datos? Es sabido que el solo procesamiento estadístico, es decir, la búsqueda de correlacio- 
nes, de regresiones, de varianzas, etc. a partir de tecnología de minería de datos será insuficiente. Estos negocios tienen ante sí una oportunidad fantástica de llegar a comprender profundamente a su comprador, a su cliente, y tal vez a su consumidor, dentro de sus entornos de hogar, del trabajo, de la vida social. Este trabajo podría tomar años, tal como tomó el Experimento de Hawthorne, pero, por otro lado, podría hay la posibilidad de hacerse en un tiempo breve si es que se llegara a usar toda la potencia de la tecnología de procesamiento de información combinado con el de modelamiento heurístico que existen hoy en día.

\section{Estudio de los procesos de innovación tecnológica en Administración}

A nivel del análisis de los obstáculos para el desarrollo de los países pobres, una de los que se menciona con persistencia en la literatura especializada es que nuestros países no tienen capacidad siquiera de adaptación tecnológica. Es decir, no tenemos la capacidad o la velocidad para usar los productos tecnológicos patentados en los países desarrollados en bien del desarrollo social o económico de nuestras naciones. Por ejemplo, la computadora, el televisor, el internet, etc. llegaron a nuestros países, pero no llegaron a tener usos intensivos e imaginativos para atacar la pobreza. El método de investigación antropológica de inmersión etnográfica, nos podría permitir comprender no solamente las causas de dicha inhibición inventiva, sino además nos podría modelar el contexto en que los peruanos desarrollan su modus vivendi, el significado que tal o cual invento tendría en sus vidas, y cómo ellos lo interpretan y qué importancia les dan para resolver sus problemas diarios. Ese conocimiento podría constituir un buen punto de partida para generar una estrategia de adaptación de innovación tecnológica.

\section{Una propuesta de niveles del conoci- miento administrativo y las tareas de investigación.}

A modo de ejercicio taxonómico hipotético tal vez se podría afirmar que el gran universo de teorías, principios, modelos, técnicas o prácticas administrativos podrían ser agrupados en tres diferentes categorías generales de conocimiento:
1. Conocimiento administrativo de aplicación universal

2. Conocimiento administrativo de aplicación particular

3. Prácticas gerenciales particulares

El conocimiento gerencial de aplicación universal incluye todo el cuerpo teórico y doctrinario de la Administración que va desde las teorías clásicas hasta las modernas incluyendo todas sus variantes tecnológicas. En este grupo tecnológico se encuentran: marketing, finanzas, riesgos, transportes, logística, modelos de manejo de personal, modelos de investigación de operaciones, planeamiento estratégico, sistemas de aseguramiento de la calidad, modelos de análisis, de pronósticos, de control operacional, o gerencial, gerencia del conocimiento, administración por objetivos o Balanced Scorecard, etc. Este es un cuerpo de conocimiento formulado y descubierto en los países desarrollados (Norteamérica y Europa) y aparentemente en dicho medio es totalmente aplicable a satisfacción plena, salvo el natural efecto de obsolescencia que las teorías también han sufrido después de un siglo de vigencia de dichas teorías o modelos. En América Latina, este conocimiento ha sido difundido y aceptado en su totalidad a fardo cerrado, sin cuestionamiento alguno y sin preguntarnos si el mismo tendrá necesidad de ajustes por tratarse de realidades (idiosincrasia, nivel educativo, historia, nivel socio económico, etc.) de países diferentes. Las universidades y los promotores de la enseñanza de la Gerencia en nuestros países han tomado dicha literatura a pie juntillas, de tal modo que en las clases repetimos a los autores originales como verdades universales inmutables.

Ahora en lo referente al conocimiento puramente tecnológico, todo pareciera indicar que efectivamente este acervo de conocimiento administrativo constituye una fuente inagotable de eficiencia en todo tipo de organizaciones, indistintamente de su sector, o nacionalidad.

El conocimiento gerencial de aplicación particular es aquel que tiene que ver esencialmente con la competencia gerencial de dirección del recurso humano, con el ejercicio del estímulo al talento gerencial para conducir una organización hacia su misión u objetivos. Den- 
tro del conocimiento de aplicación particular se podría incluir a aquel que se relaciona con el comportamiento de los agentes económicos como consumidor, como cliente, como gobierno local o nacional. Por ejemplo, una ama de casa de una zona emergente de Lima va al supermercado varias veces a la semana, lo que no ocurre con un cliente de zonas tradicionales del mismo Lima que disponen de dicho servicio desde hace décadas. Por otro lado, compradores de diferentes países tienen un comportamiento determinado frente a los estímulos publicitarios o de promoción, o los clientes de las instituciones financieras. Por ejemplo, los gerentes financieros peruanos afirman que los clientes aymaras (región de Puno) tienen las más bajas tasas de morosidad debido a su alto sentido de cumplimiento de adeudos, ya que es parte de su prestigio personal, muy cuidado por ellos. A este conocimiento podríamos llamarlo ciencia en pequeño o "petit ciencia".

Las prácticas gerenciales particulares son aquellas que nacieron y se desarrollaron con la experiencia de un gerente o grupo de gerentes, la misma que puede haberse convertido en política de trabajo, e enfoque para tomar decisiones o para resolver conflictos. Estas son precisamente las más recónditas y esquivas, son tácitas, no están escritas, porque pueden constituir la ventaja competitiva de dicha empresa o sector. Este conocimiento por ser de naturaleza y propiedad particular no constituye ciencia, ya que la ciencia por definición es pública, y está sujeta a debate abierto.

La tarea que resulta para la academia (universidades) es por un lado preparar una batería epistemológica para los tres propósitos: primero para confirmar la bondad de las teorías generales, para construir modelos locales de comprensión de los agentes económicos en todos sus procesos: laborales, mercantiles, financieros, etc. Segundo, en lo que podríamos llamar ciencia pequeña o local. Tercero, finalmente, requerimos hacer, hasta donde sea posible, un levantamiento o inventario de las prácticas gerenciales particulares en América Latina. Es decir, hacer un inventario de las prácticas gerenciales para determinar cuáles prácticas están generalizadas y podrían constituir una especie de pre-ciencia administrativa.

\section{CONCLUSIÓN}

La Administración es una ciencia que contiene teorías, tecnologías y prácticas. Como campo de investigación, tiene esencialmente dos grandes problemas: primero, que ha sido impelida tanto por las universidades como por los organismos de promoción del desarrollo de la ciencia al uso de la epistemología positivista o cuantitativa, tal y como si fuera una ciencia natural; segundo toca estudiar la aplicabilidad a todo lo descubierto y creado en los países desarrollados a la realidad de Latinoamérica, sobre todo porque la realidad social de este subcontinente tiene su propias peculiaridades. En estos esfuerzos puede ayudar mucho la Antropología Social, con el uso de sus métodos de investigación esencialmente cualitativos, sobre todo para el inventario y modelamiento de las prácticas gerenciales latinoamericanas, como semillero de nuevas teorías administrativas.

\section{REFERENCIAS BIBLIOGRÁFICAS}

Bunge, Mario (2014). "La Ciencia. Su método y su Filosofía”. Penguin Random House. Amazon.

David, Fred R. (2003). "Conceptos de Administración Estratégica”. 9ạ. Edición. Pearson. Prentice Hall. México.

Descartes, René (2014). “Descartes: The Essential Collection". Amazon Digital Services. Kindle books.

Durkheim, Emile (2013). "The Division of Labor in Society”. Second edition. McMillan.

Fayol, Henri (1987). “Administración Industrial y General”. Editorial El Ateneo. 14ª edición. Buenos Aires.

Goosen, Rick (2004). "Managers \& MBA`s: an interview with Henry Mintzberg". Journal of Business Strategy. October.

Herrnstein, Richard J y Charles MURRAY (1996). "The Bell Curve. Intelligence and Class Structure in American Life". Free Press Paperbacks. Simon and Schuster. New York.

Hidalgo, Augusto (2013). "El Componente Metafísico de las Teorías Administrativas". Revista Gestión en el Tercer Milenio vol. 16 N 31. Facultad de Ciencias Administrativas Universidad Nacional Mayor de San Marcos, Lima. Perú.

Jenofonte (1990). “Conversations of Socrates". Penguin Classics. 
Jiménez Nieto, Juan Ignacio (1981). “Tratado de Teoría Administrativa”. TECNOS. Madrid. 1981

Kaplan, Robert S. y David P. NORTON (1996). "The Balanced Scorecard". Amazon Digital Services.

Malinowsky, Bronislaw (1948). "Magia, Ciencia y Religión”. Planeta Agostini.

Maslow, Abraham (2012). "A Theory of Human Motivation". Start Publishing LLC. Kindle book.

Mcgrath, James y Bob BATES (2013). "The Little Book of Big Management Theories, and how to use them". Pearson. Kindle digital.

Mintzberg, Henry. "Managers not MBA's. A Hard Look At The Soft Practice Of Managing And Ma- nagement Development Managers Not Mbas". Kindle book.

Popper, Karl R. (1985) "La Sociedad Abierta y sus Enemigos". Paidós. Madrid.

Taylor, Frederick Winslow (1987). "Principios de las Administración Científica". Editorial El Ateneo, 10aㅡ edición. Buenos Aires.

Von Hayek, Friederich (1974). "La Pretensión del Conocimiento". Discurso al recibir el Premio Nóbel de Economía. http://www.eumed.net/ cursecon/textos/hayek_conocimiento.htm

Webb, Richard (2015). "Para conocernos mejor". Diario El Comercio. 03 agosto 2015. 
